International Journal of Engineering \& Technology, $7(2.13)(2018) 220-225$
International Journal of Engineering \& Technology
Website: $w$ ww.sciencepubco.com/index.php/IJET
Research Paper

\title{
3 D scanner integration with product development
}

\author{
Lalit Kumar $^{1}$ *, Mohd Shuaib ${ }^{2}$, Qamar Tanveer ${ }^{2}$, Vineet Kumar ${ }^{3}$, Mohd Javaid ${ }^{4}$, Abid Haleem ${ }^{5}$ \\ ${ }^{1}$ Research Scholar, Mechanical Engineering Department, Jamia Millia Islamia, New Delhi, India-110025 H. No 536, \\ Near Badi Choppal, V.P.O. Sankhol, Tehsil Bahadurgarh, Distt. Jhajjar, State Haryana, PIN-124507, India \\ ${ }^{2}$ Research Scholar, Mechanical Engineering Department, Jamia Millia Islamia, New Delhi, India-110025 \\ ${ }^{3}$ Research Scholar, Department of Mechanical Engineering, Jamia Millia Islamia, New Delhi, India-110025 \\ ${ }^{4}$ Assistant Professor, Department of Mechanical Engineering, Jamia Millia Islamia, New Delhi, India-110025 \\ ${ }^{5}$ Professor of Mechanical Engineering, Faculty of Engineering and Technology, Jamia Millia Islamia, New Delhi, India-110025 \\ *Corresponding author E-mail: athee.lalit.2007@gmail.com
}

\begin{abstract}
Purpose: The purpose of this paper is to give a basic understanding of the working and use of 3 D Scanner in reverse engineering, shape reconstruction and product designing. It discusses the use of $3 \mathrm{D}$ Scanner in product development field and also presents a method to acquire the required data of existing component through which we can develop the new product.

Design/methodology/approach: To understand the fundamental working principle of the scanner, an algorithm is discussed which shows the data collection procedure of the scanner. The obtained data is verified with existing CAD data with the help of software, and an example is also discussed to explain the process.

Findings: This study shows that the integration of scanner technology with product development cycle will help to improve the development process of any product, as this technology provides benefits through reduction in time, money and resources.

Research limitations/implications: The speed and accuracy of the Scanner depends upon the method adopted to capture the data.

Practical implications - Reverse engineering is a process of change an existing product into a new product. Development of an existing product we required the three-dimensional data of the product. The data acquisition process is critical and time-consuming. The design expert uses different approaches to collect the necessary parameters. This study discusses one of the data acquisition technology, i.e. 3dimensional scanner.

Originality/value - In this article, we are discussing one of the original methods to show the integration of scanner technology with product development.

Keywords: 3 D Scanner; Data Acquisition; Product Designing; Product Development; Product Redesigning; Reverse Engineering; Scanner Algorithm.

\section{Introduction}

Digital 3D scanner fundamentally captures an image of object and stores it in the form of digital data by converting the surface image into billions of coordinate points [1]. In other words, one can say that it effectively converts any real 3D object into digital format (XYZ coordinates of the object) in quick succession of time. It is a device through which we can acquire digital information of any physical model which can be used for further useful work.

The purpose of this study is to understand the principle and working of a 3D scanner which can later benefit through easier usage in the field of manufacturing, design, development, surveying or testing etc. Integration of 3D scanner with product development is discussed here. The three-dimensional Scanning technology can be applied in many areas, to save time, money and material [2-5]. It can be used for digital inspection, quality purpose or may be for reverse engineering through different applications. The usage of such digital tools and technique has resulted in improvement in process planning. It is more accurate in measuring the physical model and in lesser time as compared to any other technology, hence reducing errors and mistake which can turn expensive in the later stage of development. The study shows that the cost of manufacturing design cycle can be decreased up to $70 \%$ by using 3D scanning in the development cycle.

This technology can be applied successfully in the prototype development phase where the designer has to evaluate all the possible physical prototypes to find the best. Product designed using 3D scanned data requires only one prototype as it is modelled using cloud data of the physical model. As compared to other reverse engineering technologies for recreating a product, acquiring the data from $3 \mathrm{D}$ scanners and creating CAD model provides much more accurate data in the least possible time. Product development technologies such as rapid prototyping and tooling, reverse engineering etc. are increasingly enabling fast and cheap ways to create parts directly from computer-aided design models when the production is limited to few numbers.

Earlier, to develop a new design of any existing model, the development of the prototype is done with the help of physical measurements. This process is very tedious and time-consuming, which also requires in-depth knowledge of clay lead design or foam lead design, but 3D scanner converts the existing data in much easier and faster way by generating a cloud data of the existing physical model which helps to develop the concept $[6,7]$. 3D scanning gives a head start to the developer by producing a point cloud data which eventually save time instead of designing a product from scratch in the product development stage. The process begins with scanning the physical work piece and then transferring the data into CAD software for modelling. In general, the designer works on existing component by fitting the curves into it
\end{abstract}


or around it but here components are scanned, and curves are incorporated [7] with the scanned design which results in best fit curves [8]. The process of creating CAD model by using the existing physical model or existing CAD model is known as reverse engineering or reverses modeling [9]. By incorporating reverse engineering, new components/products can be introduced in the market, with the utilisation of lesser resources and in lesser time.

The obtained cloud data of any physical model can be translated directly to the engineering phase. Converting the digital data into CAD model [10], which can be used to perform better analysis through software and simulations like finite element analysis, flow simulation, stress analysis, heat and temperature distribution etc. using different software platforms. The physical model can be manufactured directly from this data using rapid prototyping machines [11], [12].

3D scanning of the manufacturing tool can be done within the manufacturing cycle. The scanner captures the changes in the tool at the time of manufacturing. Tools are mostly manually controlled to get the desired shape and size of the final component Using digital scanning, we can scan the tool and hand movements [13] which can be modified to achieve optimisation. After modifications and simulation of the movements, we can easily transfer them to the production floor to reach the target. Another use of scanning on the shop floor production is that it can be used to study the tool wear without affecting the production. This can be used smartly to avoid the tool failure and accidents by predicting the service life and trend of the failure. At the final stage of the manufacturing, 3D scanning can be integrated with the quality system [14]. It is used as a high-speed quality check where human eyes have the limitation to scan objects moving at very high speed. The scanner reads the defective finished product and actuates the removal system for removing the defected product from the production line.

\section{Data acquisition and redesigning}

There is numerous type of data scanner available in the market. We can broadly classify them into contact and non-contact type of scanner depending upon the technique used to collect the data. However, these are subdivided into further categories. In this article, we have used a non-contact type of optical scanner to collect the data. The selection of the machine itself is a daunting task which required complete knowledge of technologies and available machine. The comparative analysis of different machine is needed to be done before finalizing any decision on any type of scanner [15]. Also, there is numerous technique available through which we can compare different machine and select the best from them. The analytical network process approach [16] is one of the technologies which can be used for this purpose as it gives good result along with sensitivity analysis to strengthen our decision on the machine and to validate the result.

The non-contact device is a convenient technology for 3D data acquisition. The time used for data acquisition is shorter than other methods. There are different types of 3D scanners available based on methods of capturing objects. These Scanners can accurately measure different sizes, through lenses of different field of view. Thus, users have to select suitable 3D scanning method for their specific needs.

Scanning is one of the simpler methods to attain 3D parameters of an object using the structured-light based on triangulation method. A grid of light is targeted (projected) on the surface of the object to get the clear image. The deformed grids on the image are used to find the 3D curvature of each existing point of the object by triangulation. The calibration of the camera and projector gives the proper distance constraints between the object and the system. Fig. $\mathbf{1}$ shows the general process flow chart of the scanner.

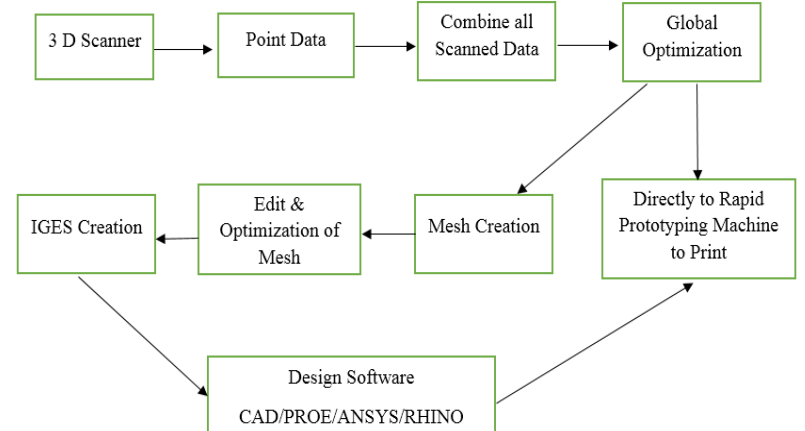

Fig. 1: Process of Data Acquisition.

The scanner used the blue light projected by the projector and collected the data with a high-resolution camera. The obtained data is in the form of a cloud of millions of points which is obtained by the scanner image. This scanned image alone is not enough to have all the required information about the scanned object, so some scans images are obtained from different angles. These images obtained from different angles have to align/merged to get better information. The aligning/merging can be done automatically or manually depending upon the requirement by providing reference points. After aligning the three-dimensional images of the object obtained which consists of almost all the information of the object dimensions and topography. This data is then globally optimised to remove the errors in the dimensions. The errors can be studied separately before and after optimisation. This data can be used to generate the three dimensional model of an object directly by using any rapid prototyping technique, or it can also be processed further.

To refine the scanned data further, we convert the scanned data into triangulation language file by creating fine meshes. These meshes are then edited and recreated again and again to get best result. This process removes the errors and holes from the scanned data which may be present due to fault in image capturing. After successfully editing and creating meshes we convert the IGES file of the scanned data. This file can now be used in reverse engineering or quality engineering for further improving or redesigning the product in any of the CAD software like PRO-E, ANSYS, and RHINO etc. ${ }^{45}$. The IGES file consists of point cloud data which can be further used to generate curves and to convert it into various solids. The conversion of the solid object from the scanned data is a lethargic process which requires time and core knowledge of the tool. The generated solid can be further used for modification according to the requirements for product development.

\section{The study of scanner}

As stated earlier, we are using a non-contact type of optical scanner for study. The optical three-dimensional digital scanning can also be classified further into different categories like laser scanner, white light scanner, computed tomography (CT) scan, time of flight scan and much more. However, we can broadly differentiate non-contact type active laser scanners into three main types according to data acquisition process, which are 1) laser triangulation scanner, 2) time of flight laser scanner and 3) phase shift scanner. We can use these techniques either independently or also we can combine them to get more versatile scanning system. However numerous other laser scanner is also available in the market which is hybrid and combinations of one or more technologies, for example, fringe interferometry.

Laser triangulation scanner is the technology in which laser is used to capture the object location and distance. The angle of reflected laser light from the source can be obtained by the sensor located in the camera, and this reflected angle can be used to obtain 3 D measurement data of the object.

Time of flight laser scanners also uses laser light to scan the object. The sensor absorbs the reflected data. A sensor detects the reflection along with the time of elapses between the emitted and 
reflected wave to detect the distance to the object (as the speed with which laser light travels is already a known quantity with high precision value).

Phase shift type laser scanners also use laser light. The scanner work by comparing the phase shift in the reflected laser light to a standard phase. This type of scanner is quite similar to the flight laser scanner. The only difference is this scanner is more accurate, and its' accuracy is further refined with the help a second scale of distance detection same as found in vernier calliper.

The scanner selection is done after the comparative analysis between different available technologies and accordingly the best suitable machine is selected as per the user requirements. The scanner selection and comparison is not discussed in this article. Here, we are discussing blue laser light scanner. The blue light works as projector beam and camera is used to capture the change in the projected light. The blue light of the projector gives an advantage of using this scanner in any light conditions (environmental) which are not possible in any other scanner.

The optical digitisation scanning system (Steinbichler Comet scanner, Germany) here is used with a powerful operating software platform (Colin 3D) for measuring systems, making photogrammetry measurements as well as editing and analysing the acquired data. The software platform is integrated with the machine hardware, and it is so designed to achieve maximum easiness in use. Also, the Colin 3D software spares us the hassle of setting countless parameters, allowing us to concentrate on the task at hand. Here a brief overview of the scanning process and the optimising process of the scanned data is described step by step. The

Table 1 below shows the specification of the scanner and the Fig. 2 shows the actual scanner.

The following steps are necessary before starting the measurement with Colin 3D:

a) Make the connections of the device correctly with hardware configuration

b) Check and install Colin 3D software if required

c) Import all the configuration files which are required to configure the hardware components with the software

d) Calibrate the device for the measurement with the standard calibration procedure

Table 1: Description of Scanner

\begin{tabular}{ll}
\hline Specification & Description \\
\hline Weight & $2.85 \mathrm{Kg}$ \\
Technology & Blue light Scanner \\
Scan Angle & 360 Degree with rotary table \\
Fastest Scanning time & 2 Seconds \\
Communication & Tripod Sensor stand and cables \\
Image Resolution & $2448^{*} 2050$ (5 Mega Pixel) \\
3 D point distance & $75 \mu \mathrm{m}$ \\
Measurement Volume & 183520 (For 75 field of view in mm3) \\
& 7826000 (For 250 field of view in mm3) \\
\hline
\end{tabular}

\section{Scanning methodology}

\subsection{Conditions of the environment during scanning}

It is necessary to keep the basic parameters of the surroundings unchanged like temperature, humidity and lighting. Any change of the temperature or humidity can cause a change in the dimensions of the scanned object as well as a change. Lighting arrangement in the place of the scanning affects the calibration of the scanner as well as on the scanning itself. A change of lighting during scanning can cause a poor quality scanning. With scans using the Steinbichler Comet, given its different technology, it does allow a change of parameters during scanning.

\subsection{Preparation of the scanned object}

Thermal stabilisation of the part to be scanned is necessary, for this the object is put in the environment where scanning has to take place to get thermal stability. Shiny objects also need treatment through some spray or powder.Positional markers are put on the job, and also on the surroundings. Their density and placement depend on the shape and size of the scanned object. The tabs are placed in such a way that an irregular triangle mesh gets created.

\subsection{Calibration of the 3D scanner}

Before making each measurement, or making a group of measurements, it is important first to perform a calibration or verification of the $3 \mathrm{D}$ scanner. In the case of an optic 3D scanner, this consists of scanning a calibration board at different distances and inclinations.

\subsubsection{Setting the input parameters of the 3D Scanner}

In the case of Steinbichler Comet 3D Scanners, we set the parameters for lighting the object during the scanning process, such that no overexposure or underexposure occurs.

\subsubsection{Scanning}

Steinbichler 3D Scanner, after scanning the designed specimen creates the resulting point clouds by assembling selfrotated/shifted scans. The reconstructed point clouds obtained after scanning contain a multitude of artefacts from reflections or the scanned surrounding surfaces.

\subsection{Export of data}

It is possible to export the raw data using several file formats, mostly *.STL format is adopted due to the universality of its use.

\subsection{Editing of data}

The exported data also contains unnecessary data errors in the surface of the triangle mesh (tiny openings, areas with a faulty normal), which need to be removed or repaired. This is most often done through independent software serving for the modification of such data. For data correction and preparation we are using INUS Rapid form XOR2. The repaired data are again exported into STL file and load in the visualisation and measuring software Volume Graphics VG Studio MAX 2.2.

\subsection{Evaluation of obtained data}

The surface mesh obtained is interpreted in the Volume Graphics through VG Studio MAX 2.2 software. For the evaluation, 23 characteristics were selected, including $\mathrm{x}$ dimensions $\mathrm{x}$ deviations in shape and orientation.

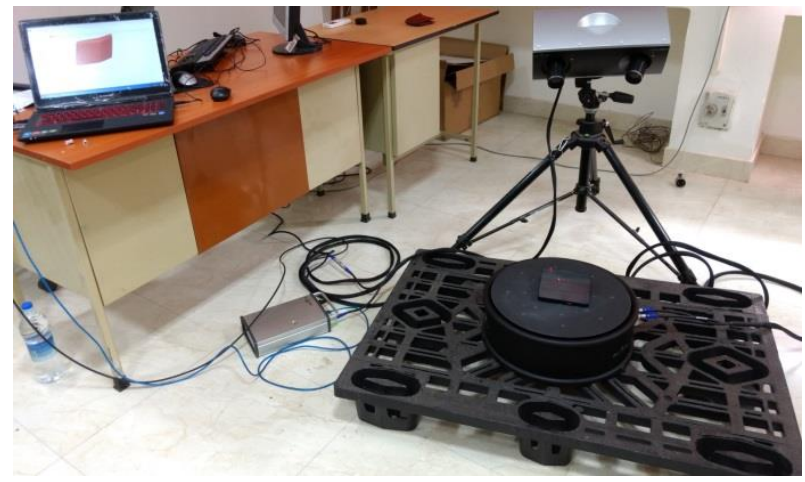

Fig. 2: The Actual 3 Dimensional Scanner.

\subsection{Challenges in data processing}

The major challenges in data processing are shown as follows: 
a) The errors in the captured data from the scanner vary with the change in the working environment temperature.

b) Whenever the lens of the projector and camera is changed due to the size of the object, the calibration changes.

c) The problem mentioned in above points, make it necessary to calibrate the scanner.

d) Aligning the obtained cloud data from two different captured images by providing one common reference points for both scan images is needed.

e) Controlling the unaligned measurement from a set of images to reduce the errors.

f) Controlling the holes and controlling the mesh size and shape as per requirements.

g) Exporting the file to any other software and handling the data is challenging.

\section{Proposed method of data collection}

To better understand the complete process of the data collection with the help of a scanner, here a simple algorithm is discussed which shows the working principle of the scanner. The scanner emits blue light onto the object through the projector, and a highresolution camera detects the reflected light from the object.

Fig. 3 shows the overview of the scanning process to calculate the dimensional parameters of the object. Let an object is placed at the location $\mathrm{O}$ as shown in Fig. 3 below. The point $\mathrm{P}$ represents projector position, and point $\mathrm{C}$ represents the camera position in Fig. 3. Let $\mathrm{f}$ is the focal length of the camera, and the projector and D are the distance between the camera and the projector which is already known and these values are calibrated in the calibration process of the scanner. Also, there is a device in the scanner to measure the incident angle of the projector beam and the reflection angle of the camera. The object $\mathrm{O}$ is placed on the rotary table to obtain $360^{\circ}$ views. The advantage of the using the rotary table is that it helps to rotate the object at an equal number of the interval so that the image can be taken at even internal. Let the object is placed in $\mathrm{XY}$ plane, and axis $\mathrm{XX}$ is parallel to projector line $\mathrm{D}$ and plane $\mathrm{XZ}$ is parallel to the camera image plane. $\mathrm{L} 1$ and $\mathrm{L} 2$ show the projection of projector line.

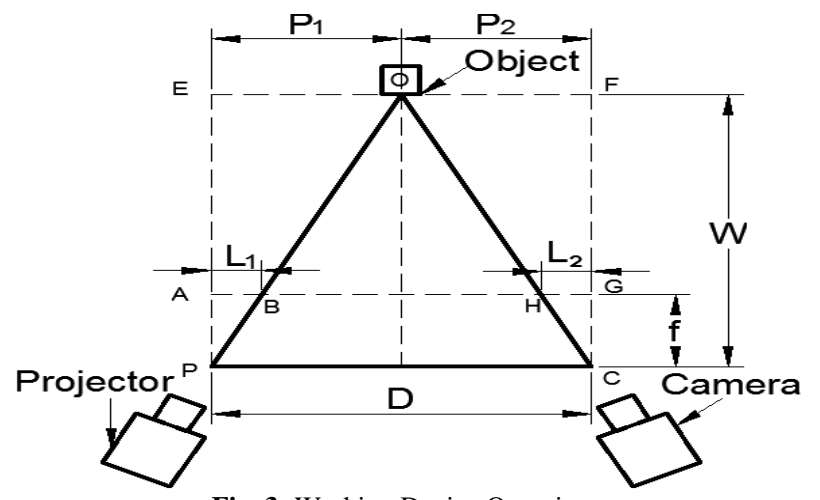

Fig. 3: Working Device Overview.

Now using simple mathematical relationships for similar triangles $(\triangle \mathrm{PAB} \& \triangle \mathrm{PEO})$ we have:

$\frac{L_{1}}{P_{1}}=\frac{f}{W}$

Similarly in $\triangle \mathrm{CHG} \& \triangle \mathrm{COF}$

$\frac{\mathrm{L}_{2}}{\mathrm{P}_{2}}=\frac{\mathrm{f}}{\mathrm{W}}$

So we have

$\frac{L_{1}}{P_{1}}=\frac{f}{W}=\frac{L_{2}}{P_{2}}$
Also from Fig. 3 we know $\mathrm{D}=\mathrm{P}_{1}+\mathrm{P}_{2}$

Therefore

$\frac{\mathrm{L}_{1}}{\mathrm{P}_{1}}=\frac{\mathrm{L}_{2}}{\mathrm{D}-\mathrm{P}_{1}}$

$\mathrm{DL}_{1}-\mathrm{L}_{1} \mathrm{P}_{1}=\mathrm{P}_{2} \mathrm{~L}_{2}$

$\mathrm{P}_{1}=\frac{\mathrm{DL}_{1}}{\mathrm{~L}_{1}+\mathrm{L}_{2}}$

Now we can calculate the coordinates position (X, Y, and Z) of the object:

$\mathrm{Z}=\frac{\mathrm{fP}_{1}}{\mathrm{WL}_{1}}=\frac{\mathrm{fD}}{\mathrm{L}_{1}+\mathrm{L}_{2}}$

$X=\frac{L_{1} Z}{f}=\frac{D L_{1}}{L_{1}+L_{2}}$

$Y=\frac{Y_{1} W}{f}=\frac{D Y_{1}}{L_{1}+L_{2}}$

Similarly, we can have the position of every point of the captured data. Then the object is rotated by a new angle, and then new data is caught in the same way. Aligning this data with previously obtained data will give the complete information of the object. In the same way, the process is repeated and again till the whole object is get scanned.

\section{Case study}

The following example shows that the digital scanner technology gives good accuracy in shape reconstruction. Some difficulties were faced, but satisfactory results were obtained. This obtained scanned data of the physical product is verified/compared with existing CAD data with the help of inspecting plus software (we are not discussing that part here). This software shows the error between the scanned data and the $\mathrm{CAD} / \mathrm{Standard}$ data.

Examples: Small Gear: Error! Reference source not found.) Gear Scan Data, Error! Reference source not found.) Post Processed Data, Error! Reference source not found.) Data Reconstruction, Error! Reference source not found.) Modelled Gear Teeth, Error! Reference source not found.) Comparison of scanned data and CAD data, Error! Reference source not found.Error! Reference source not found.) Result of comparison.

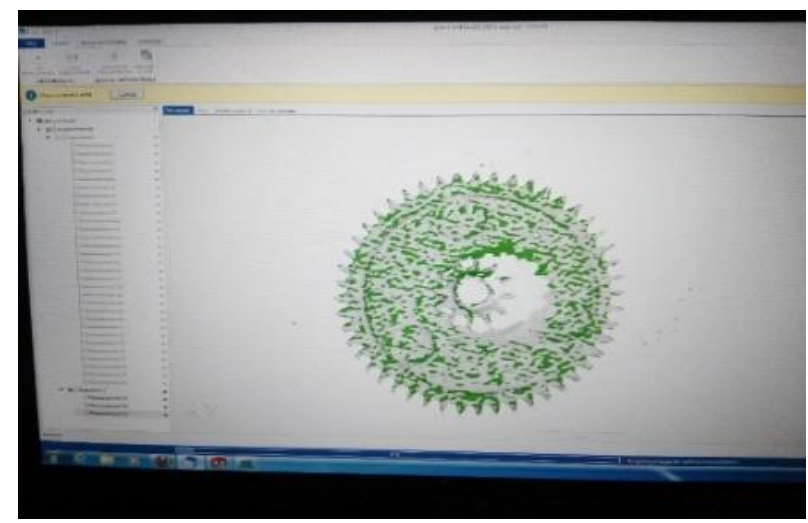

Fig. 4: A) 


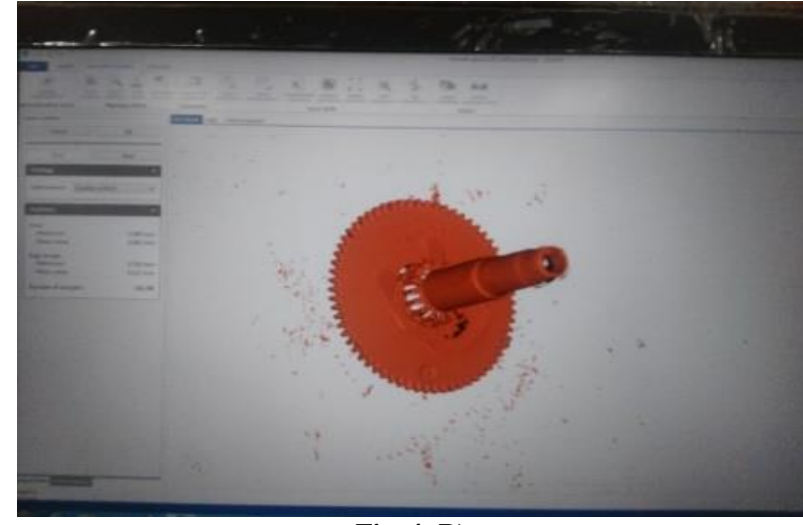

Fig. 4: B)

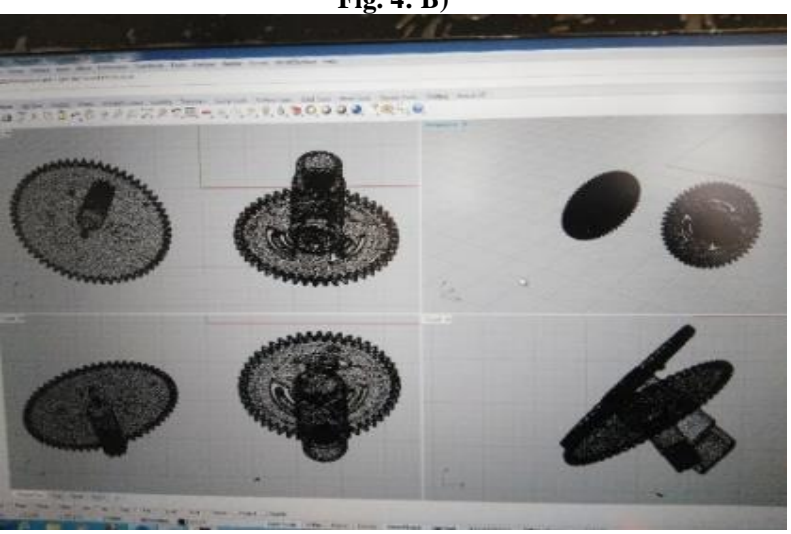

Fig. 4: C)

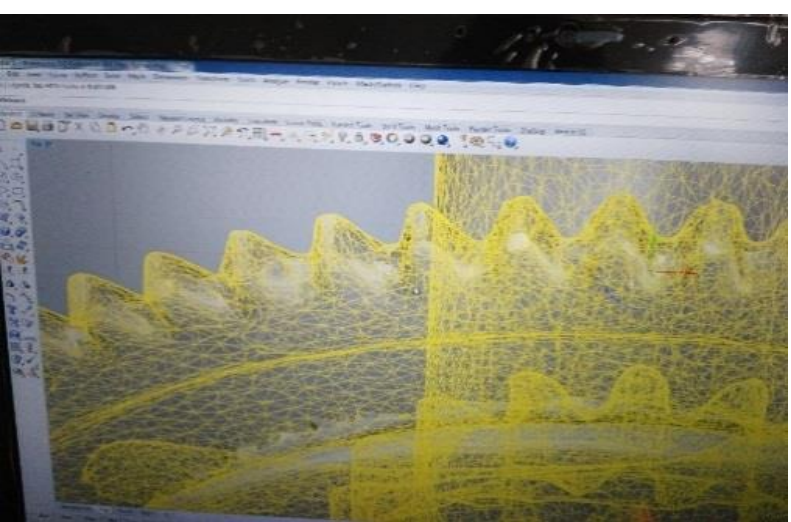

Fig. 4: D)

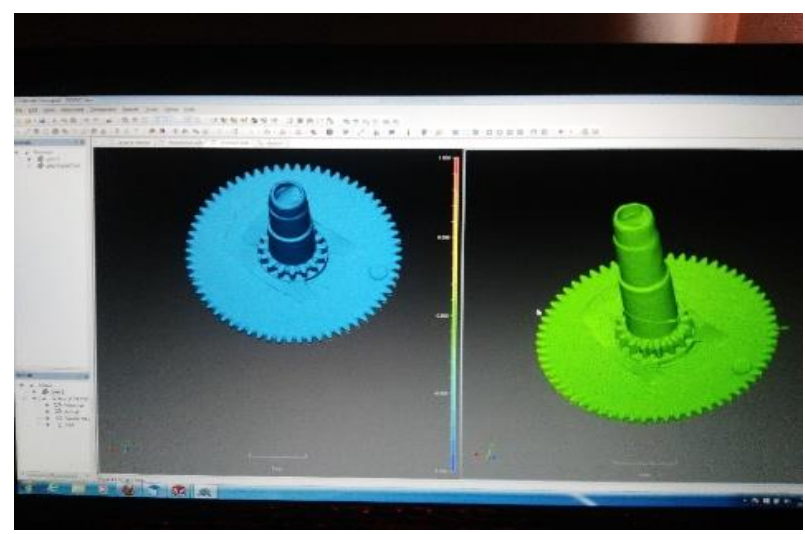

Fig. 4: E)

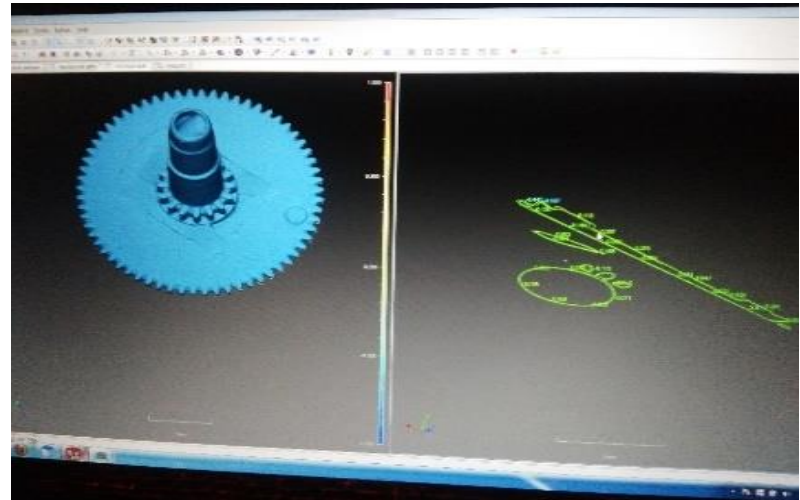

Fig. 4: F)

\section{Conclusion and future work}

This paper discussed the basic working and use of 3 D Scanner in reverse engineering, shape reconstruction and product redesigning. The digital scanning technology is growing very fast, and it is also changing the design to manufacturing cycle. This technology saves the time and cost of product development by a considerable amount which is a very critical factor in launching a new product in the market. Today, many of the companies are utilising the digital technology to acquire the rough data and also to compare the finished component with the desired specifications. Like most new technology, the digital scanning technology also saves resources like money, material, time etc. in almost every process of manufacturing from designing phase to the production phase including shipping, inspection, quality control etc. It unlocks the physical information of the object and converts it into useful digital data which has several applications. This technique is also known as 'physical to $\mathrm{CAD}$ ' or 'physical to digital' process. The complete process and working of the scanner can be easily understood by Fig. 4. The major advantages of this technology are listed below:

- It converts physical measurements data of the object into digital measurement data very accurately.

- It saves lots of time in almost all the manufacturing processes.

- It helps in removing errors in part fitting together.

- It captures engineering data and helps in optimisations.

- It helps in the modernisation of manufactured parts through $\mathrm{CAD}$ without manufacturing.

- Compare "as-designed" model to "as-built" conditions of manufactured components.

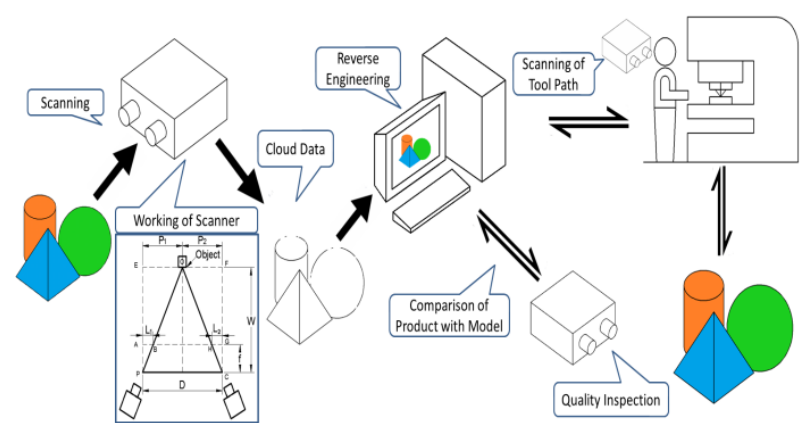

Fig. 4: The Whole Process of Scanning.

The accuracy of the scanning can be improved by increasing the number of points to be processed, which can also be achieved by using a camera of higher resolution. The problems related to precision can be sorted by focusing the camera on the borders of the image. Besides corner detection, edge detection can be performed to find complete lines across the object. If the curvatures are present in the object, they create a problem while scanning, so improvement is needed for this problem. A different position of the camera or the projector could be a solution to this issue. Another 
possibility to improve the accuracy is to increase the intensity of grid and precision of projected lines onto the object. The speed and accuracy of the scanner depend upon available method to capture the data and the accuracy of the scanned data which can be increased by increasing the number of points processed by the camera. This can be achieved by using higher resolution camera and also it can be improved by taking more scanned data again and again with more number of positions and at different angles. Development of new modelling software with a better platform and graphic user interface, integrating the cloud data with $\mathrm{CAD}$ model which can ease the working of the users. Easy removal of noise in the cloud data which can improve the modelling time resulting in a reduction in reverse engineering process time. ing a 3-D laser scanner. IEEE Trans Med Imaging. 2006; 25(6):742-754. doi:10.1109/TMI.2006.873624.

\section{References}

[1] Genta G, Minetola P, Barbato G. Calibration procedure for a laser triangulation scanner with uncertainty evaluation. Opt Lasers Eng. 2016; 86:11-19. doi:10.1016/j.optlaseng.2016.05.005.

[2] Asahina D, Taylor MA. Geometry of irregular particles: Direct surface measurements by 3-D laser scanner. Powder Technol. 2011; 213(1):70-78. doi:10.1016/j.powtec.2011.07.008

[3] De-hai Z, Yan-qin L, Gui-zhong X, Yong Y, Liang-wen W. Reverse engineering application of 3D optical scanning technology. J Appl Opt. 2015; 36(4):519-525. doi:10.5768/JAO201536.0401005.

[4] Khosravi M., Mosaddeghi F., Oveisi, M., khodayari-b, A., Aerodynamic drag reduction of heavy vehicles using append devices by CFD analysis, Journal of Central South University, Volume 22, 2015, pp 4645-4652

[5] Rashidizad H, Rahimi A. Building Three-Dimensional Scanner Based on Structured Light Technique Using Fringe Projection Pattern. J Comput Inf Sci Eng. 2014; 14(3):35001. doi:10.1115/1.4027213.

[6] Page D, Koschan A, Abidi M. Methodologies and Techniques for Reverse Engineering-The Potential for Automation with 3-D Laser Scanners. In: Raja V, Fernandes JK, eds. Reverse Engineering. London: Springer London; 2008:11-32. Doi: 10.1007/978-1-84628856-2_2.

[7] Emde M, Rossmann J. Validating a simulation of a single ray based laser scanner used in mobile robot applications. In: ROSE 2013 2013 IEEE International Symposium on Robotic and Sensors Environments, $\quad$ Proceedings. ; 2013:55-60. doi:10.1109/ROSE.2013.6698418.

[8] Paoli A, Razionale A, Barone S, Maggini M. A robotic system for 3D optical scanning of large surfaces. In: Innovative Developments in Design and Manufacturing. CRC Press; 2009:145-150. doi:10.1201/9780203859476.ch20.

[9] Dhillon DS, Govindu VM. Geometric and radiometric estimation in a structured-light 3D scanner. Mach Vis Appl. 2015; 26(2-3):339352. Doi: 10.1007/s00138-015-0667-0.

[10] Kumar L, Kumar V, Haleem A. Rapid Prototyping Technology for New Product Development. IJISET - Int J Innov Sci Eng Technol. 2016; $3 \quad$ (1):287-292. http://ijiset.com/vol3/v3s1/IJISET_V3_I1_39.pdf.

[11] Kumar L, Tanveer Q, Kumar V, Javaid M, Haleem A. Developing low cost 3 D printer. Int J Appl Sci Eng Res. 2016; 5(6):433-447. doi:10.6088/ijaser.05042.

[12] Khodayari Bavil, A., Razavi, S.E., on the thermo-flow behavior in a rectangular channel with skewed circular ribs, Mechanics \& Industry, 182 (2017) 225, https://doi.org/10.1051/meca/2016057.

[13] Yang B, Zang Y. Automated registration of dense terrestrial laserscanning point clouds using curves. ISPRS J Photogramm Remote Sens. 2014; 95:109-121. doi:10.1016/j.isprsjprs.2014.05.012.

[14] Wang H, Wang C, Luo H, et al. Object detection in terrestrial laser scanning point clouds based on hough forest. IEEE Geosci Remote Sens Lett. 2014; 11(10):1807-1811. doi:10.1109/LGRS.2014.2309965.

[15] Ilyas IP. 3D Machine Vision and Additive Manufacturing: Concurrent Product and Process Development. IOP Conf Ser Mater Sci Eng. 2013; 46(1):12029. doi:10.1088/1757-899X/46/1/012029.

[16] Kovacs L, Zimmermann A, Brockmann G, et al. Accuracy and precision of the three-dimensional assessment of the facial surface us- 